\title{
Variaciones diarias de la oferta forrajera, efecto sobre la producción y calidad de la leche
}

\author{
Daily variations of forage allowance, effects on milk production \\ and quality
}

\begin{abstract}
César Mendoza F, ${ }^{1}$ Zoot, Martha Pabón R, ${ }^{1,2}$ Ph.D, Juan Carulla F, ${ }^{1 *}$ Ph.D.
${ }^{1}$ Universidad Nacional de Colombia, Facultad de Medicina Veterinaria y de Zootecnia, Departamento de Ciencias para la Producción Animal, Grupo de Investigación en Nutrición Animal. '2Departamento de Química, Facultad de Ciencias. Bogotá, Colombia. *Correspondencia: jecarullaf@bt.unal.edu.co.
\end{abstract}

Recibido: Diembre de 2010; Aceptado: Agosto de 2011.

\section{RESUMEN}

Objetivo. Determinar el efecto de las variaciones de la oferta diaria de forraje sobre la producción y calidad de la leche. Materiales y métodos. Determinando el área de oferta diaria mediante un sistema de posicionamiento global y empleando la técnica de doble muestreo para medir producción de biomasa previa y posterior al pastoreo, se estimó su consumo diario por diferencia agronómica. Se estimaron variaciones dentro y entre fincas para los parámetros evaluados. Se estableció el grado de asociación entre las variaciones diarias en la oferta con el consumo, el volumen y calidad de la leche. Resultados. Los resultados evidenciaron asociación entre variaciones de oferta diaria y consumo de forraje $\left(R^{2}=0.65\right)$ y la variación en volumen diario de leche $\left(R^{2}=0.45\right)$. Las variaciones en grasa no mostraron relación con cambios en la oferta $\left(R^{2}=0.0\right)$ y sus valores fueron asociados a la relación forraje/suplemento $\left(R^{2}=0.52\right)$. Las variaciones en proteína tuvieron menor asociación con las variaciones en la oferta $\left(R^{2}=0.17\right)$ y mayor con el consumo de materia seca $\left(R^{2}=0.34\right)$. Conclusiones. Los resultados sugieren que variaciones en la oferta forrajera explican en mayor grado las variaciones en el consumo de materia seca con un efecto directo sobre el volumen de leche y en menor grado sobre las variaciones en su composición.

Palabras clave: Consumo, forraje, oferta forrajera, pastoreo en franjas (Fuente: CAB). 


\section{ABSTRACT}

Objective. To determine the effects of daily forage allowance variations on production and milk quality. Materials and methods. This study took place in five commercial farms and included two periods of observation. The daily forage intake was estimated through agronomic difference, measuring the daily area of allowance with a GPS and using the Doubled Sampling Technique to estimate biomass yield and the remaining herbage after pasture defoliation. Variations for each parameter were estimated within and between farms in order to determine its magnitude, relationships between daily herbage allowance and forage intake, and its influence on milk yield and composition during both periods of observation. Results. There was an association between daily herbage allowance and variation on daily forage intake $\left(r^{2}=0.65\right)$ as for variation on daily milk yield $\left(r^{2}=0.45\right)$. Daily variation on milk fat showed no direct relationship with daily herbage allowance, but its values were associated with forage to supplement ratio $\left(r^{2}=0.52\right)$. Relation between daily variation of milk protein and daily herbage allowance was low $\left(r^{2}=0.17\right)$, but higher between protein concentration and forage dry matter intake $\left(r^{2}=0.34\right)$. Conclusions. The results suggest that variation on daily herbage allowance explained largely the variations on dry matter intake, leading to a direct effect on milk yield, but explain little the variations on milk fat and milk protein.

Key words: Consumption, forage, intake, strip grazing (Source: $C A B$ ).

\section{INTRODUCCIÓN}

En Colombia, la lechería especializada maneja el sistema de pastoreo en franjas con cuerda eléctrica (pastoreo racional) permitiendo aumentar la carga animal a través del suministro continuo de pasto fresco, libre de pisoteo y excretas, haciendo un uso más eficiente de las praderas. Comercialmente, se maneja un escenario en que la carga animal permanece más o menos constante. Sin embargo, la cantidad de forraje disponible por unidad de área varía a través del año debido a condiciones climáticas y de manejo. Los cambios entre la carga y el forraje disponible generan variaciones en la oferta de forraje por animal así como en la productividad.

Los estudios con pasturas han mostrado que la oferta forrajera determina en gran medida el volumen de leche y que puede tener impacto sobre la concentración de proteína (1-5). Blaser et al (6) atribuyeron las variaciones en la producción diaria de leche a fluctuaciones en el consumo de materia seca, asociadas principalmente a cambios súbitos en la disponibilidad diaria de forraje, así como comportamientos propios de animales en pastoreo (selectividad, competencia, entre otros).
Estudios con ofertas controladas sugieren que las proporciones forraje/concentrado en sistemas de pastoreo pueden determinar la concentración de grasa en leche. Mayores niveles de suplementación de concentrado son asociados a mayor volumen de leche, incrementos en proteína y reducciones en la concentración de grasa (7). Si bien existe una amplia documentación sobre el efecto de la cantidad de oferta sobre la producción de leche, la información relacionada al impacto que tienen las variaciones diarias sobre la calidad composicional de la leche es menor. La mayoría de estudios que han determinado el efecto de la oferta sobre la calidad de la leche han sido realizados con ofertas controladas y constantes contrario a lo que sucede en las explotaciones comerciales donde éste parámetro no es medido dando cabida a la presentación de variaciones diarias de la misma. Adicionalmente es probable que las relaciones entre el forraje y el concentrado también varíen en tiempos muy cortos y las consecuencias sobre los componentes de la leche no se han determinado. 
El objetivo de este trabajo fue determinar la magnitud de variación de la oferta forrajera en periodos cortos de tiempo en hatos comerciales y su asociación con el consumo de forraje, volumen y calidad de la leche.

\section{MATERIALES Y MÉTODOS}

Sitio de estudio. Se seleccionaron cinco hatos comerciales ubicados en la región occidental de la Sabana de Bogotá (Colombia) que manejaban pastoreo en franjas con cuerda eléctrica. En la tabla 1 se describen algunas características generales del entorno en que se encuentra cada uno de los hatos evaluados.

En todos los hatos se realizó la rutina de ordeño dos veces al día (mañanatarde), momento en que se suministró la mayoría de suplementos, principalmente el balanceado comercial como común denominador en el manejo alimenticio de los hatos de la región.

La asignación de forraje para los hatos 1 y 2 se realizó dos veces al día inmediatamente después de cada ordeño, repartiendo el área de oferta en dos cortes similares en tamaño. Los hatos 3 y 4 presentaron por su parte una mayor frecuencia de asignación, realizando respectivamente 3 y 6 cortes equitativos durante el día. La asignación para el hato 5 se distribuyó en un $25 \%$ en la mañana y el $75 \%$ restante en la tarde.

Períodos de muestreo. La colecta y registro de la información de los hatos se llevó a cabo en 14 días repartidos en dos periodos de observación de 7 días para cada uno de ellos. El proceso tomó un total de 71 días repartidos en dos periodos comprendidos entre el 21 de Julio al 10 de Septiembre del 2008 (Periodo 1), y del 30 de Marzo a 19 de Abril del 2009 (Periodo 2).

Variables medidas. La determinación de la producción de biomasa aérea y remanente, se realizó mediante la técnica de doble muestreo (8), cuya calibración se llevó a cabo cortando a una altura de $8 \mathrm{~cm}$ desde suelo y pesando 5 marcos de $0.25 \mathrm{~m}^{2}$ de 15 colocados aleatoriamente en cada franja. A partir del muestreo de biomasa, se procedió a separar constituyentes de la pradera (gramíneas, leguminosas, arvenses y material muerto). Las muestras fueron pesadas y posteriormente llevadas a materia seca $\left(100^{\circ} \mathrm{C}\right.$ por 24 horas, según AOAC (9). La diferencia entre la biomasa total y la cantidad de material muerto determinó la cantidad de biomasa disponible por unidad de área.

La oferta forrajera diaria (kgMS/vaca, o, $\mathrm{KgMS} / 100 \mathrm{~kg}$ de PV) se estimó midiendo las áreas en oferta con un equipo de geoposicionamiento GPSMAP $®$ 76CSx (Garmin Ltda., Kansas, EUA), calculando la cantidad de biomasa disponible y determinando el número de animales en la franja de pastoreo. El peso promedio de los animales fue suministrado por los encargados de cada hato.

El consumo fue estimado por diferencia agronómica entre la producción de forraje, y el remanente una vez defoliada el área destinada para el día.

Se registró diariamente el volumen total de leche producido en cada uno de los ordeños

Tabla 1. Ubicación y características generales de los hatos involucrados en el análisis.

\begin{tabular}{|c|c|c|c|c|c|}
\hline Localización & Hato 1 & Hato 2 & Hato 3 & Hato 4 & Hato 5 \\
\hline Municipio & San Francisco & Facatativa & Funza & Facatativa & Tenjo \\
\hline Altitud, msnm & 2817 & 2745 & 2565 & 2815 & 2592 \\
\hline Equipo de ordeño & Móvil & Móvil & Establo & Establo & Establo \\
\hline Genética animal & Normando & Holstein, Red Poll, Normando, Simm* & Holstein, Ayrshire, HXA* & Holstein, $\mathrm{HxJ}{ }^{*}$ & Holstein \\
\hline Pastura 1 & $P_{C}+$ Lsp. + Nat & $P c+L s p .+D g+H I+A o T p+T r$ & $P c$ & $P c+L s p$ & $P C$ \\
\hline Suple ${ }^{2}$ & $B C, A C, L C$ & $\mathrm{BC}$ & $B C, H A, S M, A C, M T, J C, S b G, S A, C A$ & $\mathrm{BC}, \mathrm{P}$ & $\mathrm{BC}, \mathrm{SA}, \mathrm{SC}$ \\
\hline
\end{tabular}

${ }^{1} \mathrm{Ao}=$ Anthoxantum odoratum; $\mathrm{Dg}=$ Dactilis glomerata; $\mathrm{HI}=$ Holcus lanatus; $\mathrm{Lsp} .=$ Lollium $\mathrm{sp} ;$ PC=Pennisetum clandestinum; I=Trifolium pratense; Tr=Trifolium repens; Nat=especies nativas; ${ }^{2} \mathrm{AC}=$ Afrecho de Cervecería; $\mathrm{BC}=$ Balanceado Comercial; $\mathrm{CA}=$ Cascarilla de Algodón; HA=Heno de Angleton; JC=Jabónes Cálcicos; LC=Levadura de Cerveza; MT=Mogolla de Trigo; P=Palmiste; SA=Semilla de Algodón; SbG=Subproductos de Galletería; Sc=Fuente comercial de levaduras (Saccharomyces cerevisiae); SM=Ensilaje de Maíz. *Simm=Simmental; HxA=Holstein x Ayrshire; HxJ=Holstein x Jersey 
realizados en las fincas y se corrigió al $4 \%$ de grasa (10). Muestras de $50 \mathrm{ml}$ de leche fueron tomadas directamente del tanque de almacenamiento e inmediatamente refrigeradas para análisis de composición de grasa y proteína con Milk Scan $®$ (Foss Electric, Hillerd, Dinamarca) en el laboratorio de calidad de la planta procesadora de leche COLANTA ubicada en el municipio de Funza, Cundinamarca.

Análisis estadístico. Se usó estadística descriptiva con el objetivo de caracterizar los valores de producción de biomasa, oferta forrajera, consumo y desempeño productivo de los hatos seleccionados. El coeficiente de variación, la desviación estándar y los máximos y mínimos se utilizaron como medidas para determinar la magnitud de la variación diaria de los parámetros productivos con respecto al promedio en cada periodo de muestreo particular para cada hato. Para estimar la precisión en el ajuste diario de la franja, se asoció la biomasa disponible y el área asignada a través de regresión lineal.

Las variaciones de las diferentes variables en cada hato fueron estandarizadas en relación al promedio de la variable en ese hato y expresadas en porcentaje. Posteriormente, se analizó la relación entre porcentaje de variación diaria de la oferta de forraje y el porcentaje de variación diaria en el consumo, la producción de leche, deposición de grasa y proteína usando regresión lineal con el software R v2.10.0 (11). La misma metodología fue utilizada para establecer el grado de asociación entre el consumo de forraje y la relación forraje/concentrado con la calidad composicional de la leche.

\section{RESULTADOS}

\section{Composición botánica, biomasa disponible, oferta forrajera, consumo de forraje y producción de leche.} Las pasturas en su mayoría estaban constituidas por gramíneas. Solamente en una de las mediciones se encontró una pastura con leguminosas. La proporción de material muerto se encontró entre 10 y $32 \%$ de la composición de las praderas evaluadas. La menor presencia de material muerto estuvo asociada a los valores más bajos de oferta de forraje.

Se obtuvieron valores de producción de biomasa total entre $222.3 \mathrm{gMS} / \mathrm{m}^{2}$ y $527.5 \mathrm{gMS} / \mathrm{m}^{2}$. Aunque se esperaban diferencias en la producción de biomasa entre los periodos de observación debido cambios en la precipitación, ésta fue similar entre los periodos, por lo cual, en varios de los hatos, la disponibilidad de biomasa entre periodos fue similar. No obstante, algunos de los potreros donde se encontraban los hatos, presentaron áreas de encharcamiento (hatos 3 y 4 específicamente), por lo que los resultados referentes a la producción de biomasa en estos hatos mostraron diferencias entre los periodos de muestreo con valores menores en el segundo periodo monitoreado (Tabla 2).

El área asignada por vaca varió entre 23.2 y $107.4 \mathrm{~m}^{2} / \mathrm{vaca}$, correspondiendo a una diferencia de 4.6 veces entre la menor y la mayor (Tabla 2). Las variaciones en el área asignada se asociaron $\left(R^{2}=0.27, p<0.01\right)$ a las variaciones en el forraje disponible (Figura 1), por lo cual se presentó una
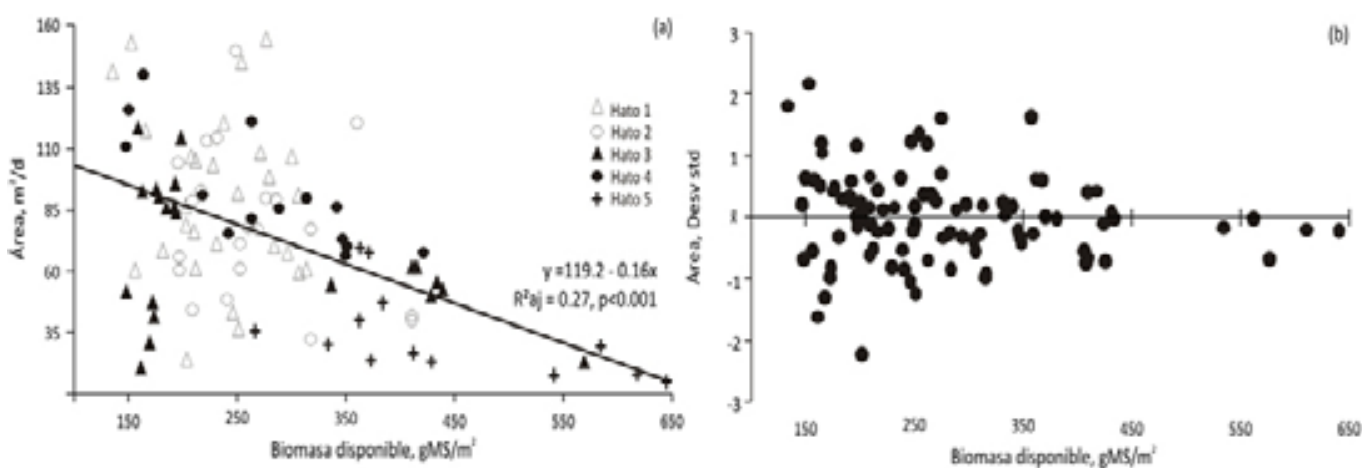

Figura 1. Relación entre la biomasa total disponible y el área de oferta diaria por animal (a), y residualidad de los datos (b). 
Tabla 2. Valores promedio de los parámetros estimados y registrados en cada hato evaluado durante siete días consecutivos en dos periodos $(P)$ de observación.

\begin{tabular}{|c|c|c|c|c|c|c|c|c|c|c|}
\hline & \multicolumn{2}{|c|}{ Hato1 } & \multicolumn{2}{|c|}{ Hato2 } & \multicolumn{2}{|c|}{ Hato3 } & \multicolumn{2}{|c|}{ Hato4 } & \multicolumn{2}{|c|}{ Hato5 } \\
\hline & P1 & $\mathbf{P 2}$ & P1 & $\mathbf{P 2}$ & P1 & $\mathbf{P 2}$ & P1 & $\mathbf{P 2}$ & P1 & $\mathbf{P 2}$ \\
\hline \multicolumn{11}{|l|}{ Composición de la pradera, \% } \\
\hline Gramínea & 100 & 95 & 66 & 97 & 100 & 100 & 100 & 100 & 100 & 100 \\
\hline Leguminosa & - & - & 25 & - & - & - & - & - & - & - \\
\hline Arvenses & - & 5 & 10 & 3 & - & - & - & - & - & - \\
\hline Material verde seco & 77 & 68 & 81 & 75 & 69 & 79 & 72 & 73 & 82 & 90 \\
\hline Material muerto & 23 & 32 & 19 & 25 & 31 & 21 & 28 & 27 & 18 & 10 \\
\hline Edad de rebrote, $\mathrm{d}$ & 60 & 62 & 90 & 70 & 25 & 23 & 39 & 45 & 51 & 50 \\
\hline \multicolumn{11}{|l|}{ Biomasa, gMS/m² } \\
\hline Total & 324 & 312 & 339 & 329 & 610 & 222 & 467 & 288 & 475 & 528 \\
\hline Disponible & 249 & 213 & 275 & 248 & 420 & 176 & 335 & 209 & 389 & 476 \\
\hline No. animales & 122 & 120 & 86 & 80 & 106 & 124 & 75 & 69 & 48 & 52 \\
\hline Área disponible, m2/vaca/d & 97.6 & 77.6 & 65.3 & 107.4 & 51.9 & 73.9 & 80.0 & 103.1 & 47.4 & 23.2 \\
\hline \multicolumn{11}{|l|}{ Oferta forrajera } \\
\hline kgMS/vaca/día & 24.3 & 16.5 & 18.0 & 26.6 & 21.8 & 13.0 & 26.8 & 21.6 & 18.4 & 11.0 \\
\hline $\mathrm{kgMS} / 100 \mathrm{kgPV}$ & 4.0 & 2.8 & 3.0 & 4.4 & 3.6 & 2.2 & 4.5 & 3.6 & 3.1 & 1.8 \\
\hline \multicolumn{11}{|l|}{ Consumo } \\
\hline Forraje, kgMS/vaca/día & 12.4 & 14.1 & 8.8 & 12.5 & 13.7 & 7.7 & 12.2 & 11.8 & 13.7 & 8.7 \\
\hline Eficiencia de pastoreo, \% & 51.2 & 85.3 & 48.8 & 47.0 & 63.0 & 59.1 & 45.7 & 54.5 & 74.1 & 78.8 \\
\hline Suplementos, kgMS/vaca/día & 6.1 & 3.6 & 1.1 & 1.2 & 9.3 & 12.1 & 6.2 & 6.2 & 4.1 & 4.1 \\
\hline Total, kgMS/vaca/día & 18.5 & 17.7 & 9.8 & 13.7 & 23.0 & 19.8 & 18.4 & 18.0 & 17.8 & 12.8 \\
\hline Forraje:Total & 0.7 & 0.8 & 0.9 & 0.9 & 0.6 & 0.4 & 0.7 & 0.6 & 0.8 & 0.7 \\
\hline \multicolumn{11}{|l|}{ Producción } \\
\hline Leche, kg/vaca/día & 12.6 & 13.0 & 11.8 & 13.3 & 22.8 & 17.4 & 20.3 & 19.9 & 18.2 & 15.0 \\
\hline Leche $4 \%$ grasa, kg/vaca/día & 12.4 & 12.9 & 11.6 & 13.1 & 20.5 & 15.5 & 18.5 & 18.6 & 17.6 & 14.0 \\
\hline Grasa, \% & 3.9 & 3.9 & 3.9 & 3.9 & 3.3 & 3.3 & 3.4 & 3.5 & 3.8 & 3.6 \\
\hline Grasa, g/vaca/día & 489 & 510 & 462 & 519 & 758 & 567 & 695 & 705 & 690 & 533 \\
\hline Proteína, \% & 3.2 & 3.2 & 2.9 & 2.9 & 3.0 & 3.0 & 2.9 & 3.0 & 2.9 & 2.7 \\
\hline Proteína, g/vaca/día & 403 & 422 & 339 & 391 & 692 & 517 & 592 & 595 & 524 & 413 \\
\hline
\end{tabular}

menor dispersión entre la oferta de forraje por vaca día que varió entre 11 y 26.8 $\mathrm{kg} / \mathrm{vaca} / \mathrm{día}$, correspondiente a 2.4 veces entre la menor y la mayor. De manera similar, se comportó la variación en la oferta expresada en KgMS/100kgPV/día (Tabla 2). La variación de la oferta entre días, dentro de un mismo hato, fue mayor en los hatos 1,2 y 3 y mas grande en la medida que disminuyó la cantidad de forraje disponible por unidad de área (Figura 1b).

El consumo de forraje estuvo entre 7.7 y $14.1 \mathrm{KgMS} / \mathrm{vaca} / \mathrm{d}$ y se encontró asociado con los cambios en la oferta $\left(R^{2}=0.49, p<0.01\right)$ con un comportamiento curvilíneo, donde a medida que aumenta la oferta el consumo se incrementó. El ascenso de la pendiente que presentó el consumo, debido a un aumento en la oferta disminuyó a medida que la cantidad de biomasa disponible aumentó (Figura 2). De igual manera, la oferta tuvo relación con la eficiencia de uso de la pradera $\left(R^{2}=0.53\right.$, $\mathrm{p}<0.01$ ), observándose que en escenarios de restricción alimenticia los animales hacen una defoliación más agresiva sobre la pastura (mayor presión de pastoreo), contrario a cuando se dispone de una mayor oferta de forraje (Figura 2).

La producción promedio vaca día entre hatos y periodos presentó valores entre 11.8 y $22.8 \mathrm{~kg} /$ día. Las diferencias en estos promedios estuvieron asociadas al consumo de MS. A medida que el consumo total de MS fue mayor, la producción de leche aumentó $\left(R^{2}=0.45, p<0.01\right)$, así como su contenido 


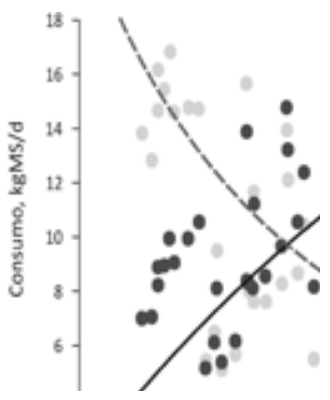

Figura 2. Relación entre la oferta forrajera, consumo de forraje y eficiencia de pastoreo.

de proteína $\left(\mathrm{R}^{2}=0.23, \mathrm{p}<0.01\right)$ con valores entre 2.7 y $3.2 \%$. Se encontraron valores de grasa entre 3.3 y $3.9 \%$, cuyas diferencias estuvieron explicadas principalmente por la relación forraje/concentrado, presentando un efecto negativo sobre el tenor graso al aumentar la proporción de concentrado en la dieta $\left(R^{2}=0.52, p<0.01\right)$.

Variaciones diarias en la oferta forrajera, consumo de MS, volumen y calidad de la leche. En las variables medidas, las mayores variaciones las presentó la oferta de forraje. El coeficiente de variación para esta variable dentro de los hatos estuvo entre $8.3 \mathrm{y}$ $37.5 \%$ de los cuales el $70 \%$ de los datos estuvieron por encima del $15 \%$ de variación. Adicionalmente, las diferencias entre los días de menor y mayor oferta dentro de la mayoría de los hatos en un mismo periodo se encontraron por encima del $50 \%$.

Los coeficientes de variación para el consumo de forraje en su mayoría ( $60 \%$ de los datos) estuvieron por debajo de $15 \%$, variaciones inferiores a las presentadas con la oferta. Sin embargo, las variaciones en el consumo dentro de cada hato y periodo expresadas con relación al promedio de consumo del periodo se relacionaron estrechamente $\left(R^{2}=0.65, p>0.01\right)$ con las variaciones en la oferta de forraje expresadas con relación al promedio de cada periodo dentro de cada hato (Figura 3).

Las variaciones en la producción de leche y su composición fueron mucho menores a las presentadas para oferta y consumo
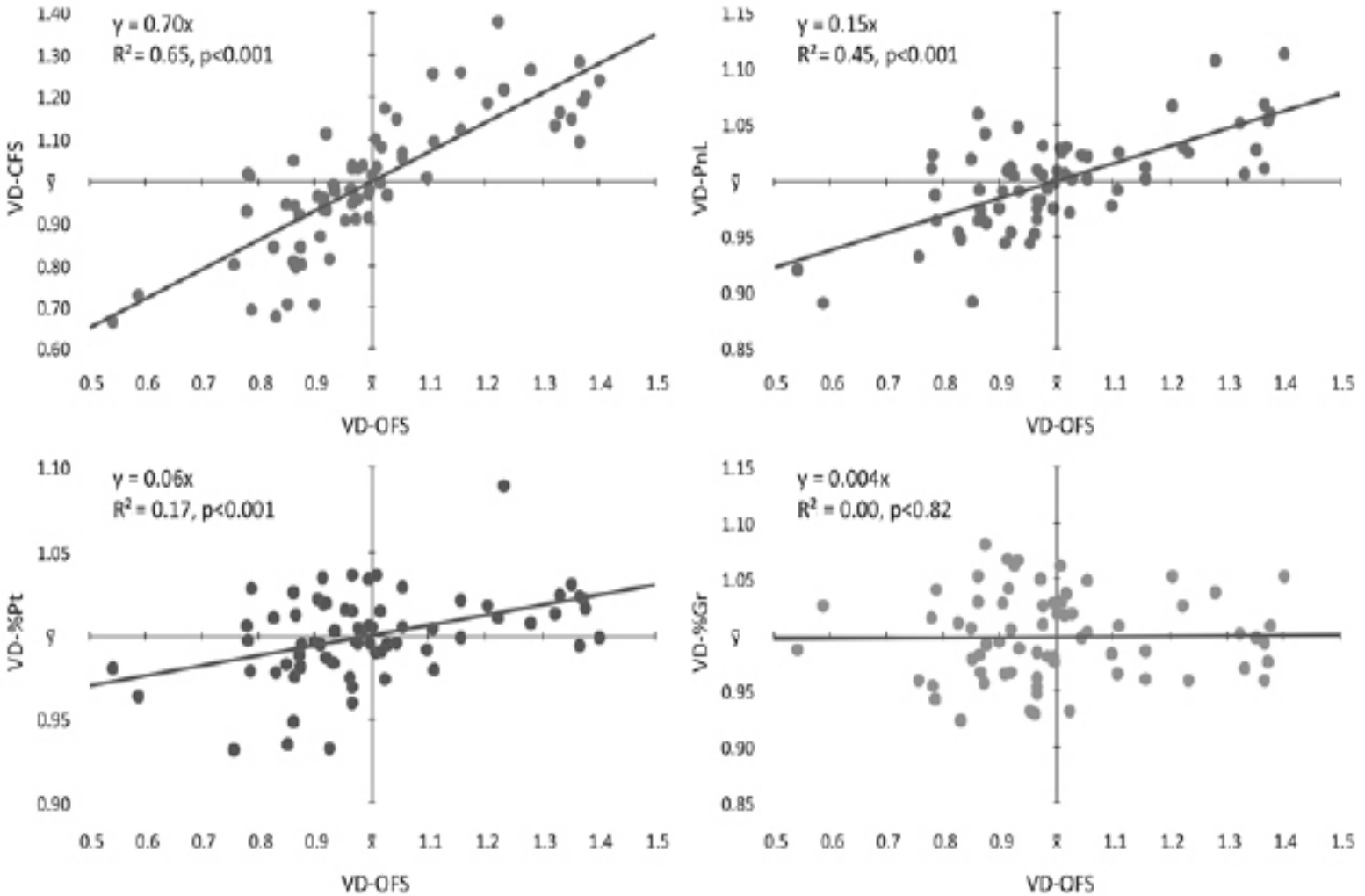

Figura 3. Efecto de la variación diaria (VD) con respecto al promedio de la oferta (OFS) sobre las variaciones diarias del consumo de forraje (CFS), producción de leche (PnL), composición de proteína (\%Pt) y de grasa (\%Gr). 
Mendoza - Variaciones diarias de la oferta forrajera

Tabla 3. Rangos y estimadores de variación de los parámetros productivos en cada hato durante los periodos de observación.

\begin{tabular}{|c|c|c|c|c|c|c|c|c|c|c|}
\hline & \multicolumn{2}{|c|}{ Hato1 } & \multicolumn{2}{|c|}{ Hato2 } & \multicolumn{2}{|c|}{ Hato3 } & \multicolumn{2}{|c|}{ Hato4 } & \multicolumn{2}{|c|}{ Hato5 } \\
\hline & P1 & $\mathbf{P 2}$ & P1 & $\mathbf{P 2}$ & P1 & $\mathbf{P 2}$ & P1 & P2 & P1 & $\mathbf{P 2}$ \\
\hline \multicolumn{11}{|c|}{ Oferta forrajera, kgMS/vaca/día } \\
\hline Max & 33.3 & 21.8 & 20.2 & 36.8 & 28.1 & 16.7 & 29.1 & 27.3 & 25.0 & 12.5 \\
\hline Min & 22.0 & 12.2 & 12.3 & 21.7 & 16.6 & 11.4 & 22.9 & 16.2 & 9.8 & 8.7 \\
\hline$\% \mathrm{CV}$ & 16.4 & 19.7 & 19.9 & 19.0 & 18.6 & 14.8 & 8.3 & 17.6 & 37.5 & 11.8 \\
\hline Desv std & 4.0 & 3.3 & 3.6 & 5.1 & 4.1 & 1.9 & 2.2 & 3.8 & 6.9 & 1.3 \\
\hline \multicolumn{11}{|c|}{ Consumo de forraje, kgMS/vaca/día } \\
\hline Max & 13.6 & 16.2 & 11.1 & 15.5 & 16.0 & 10.6 & 13.7 & 14.8 & 17.6 & 10.6 \\
\hline Min & 11.9 & 11.3 & 6.2 & 10.5 & 11.0 & 5.2 & 11.4 & 8.2 & 9.1 & 7.0 \\
\hline$\% \mathrm{CV}$ & 4.8 & 10.9 & 20.0 & 13.6 & 12.3 & 27.7 & 6.9 & 20.4 & 24.2 & 15.6 \\
\hline Desv std & 0.6 & 1.5 & 1.8 & 1.7 & 1.7 & 2.1 & 0.8 & 2.4 & 3.3 & 1.4 \\
\hline \multicolumn{11}{|c|}{ Consumo total, kgMS/vaca/día } \\
\hline Max & 19.7 & 19.7 & 12.2 & 16.7 & 25.7 & 22.5 & 19.9 & 21.0 & 21.7 & 14.7 \\
\hline Min & 18.0 & 14.9 & 7.3 & 11.8 & 20.1 & 17.7 & 17.6 & 14.4 & 13.2 & 11.1 \\
\hline$\% \mathrm{CV}$ & 3.2 & 8.7 & 17.8 & 12.4 & 7.8 & 10.2 & 4.6 & 13.4 & 18.6 & 10.6 \\
\hline Desv std & 0.6 & 1.5 & 1.8 & 1.7 & 1.8 & 2.0 & 0.8 & 2.4 & 3.3 & 1.4 \\
\hline \multicolumn{11}{|c|}{ Producción de leche $4 \%$ Gr, kg/vaca/día } \\
\hline Max & 13.0 & 13.6 & 12.9 & 14.6 & 21.0 & 15.9 & 18.7 & 19.5 & 18.8 & 14.4 \\
\hline Min & 11.8 & 12.0 & 10.4 & 12.4 & 19.7 & 14.7 & 18.1 & 17.5 & 15.7 & 13.5 \\
\hline$\% \mathrm{CV}$ & 2.9 & 4.0 & 7.9 & 5.7 & 2.5 & 3.3 & 1.3 & 4.2 & 7.1 & 2.6 \\
\hline Desv std & 0.4 & 0.5 & 0.9 & 0.7 & 0.5 & 0.5 & 0.2 & 0.8 & 1.2 & 0.4 \\
\hline \multicolumn{11}{|l|}{ Grasa, \% } \\
\hline Max & 4.1 & 4.1 & 4.1 & 4.1 & 3.5 & 3.5 & 3.7 & 3.8 & 4.0 & 3.8 \\
\hline Min & 3.6 & 3.8 & 3.7 & 3.6 & 3.1 & 3.0 & 3.3 & 3.3 & 3.6 & 3.4 \\
\hline$\% \mathrm{CV}$ & 4.8 & 3.0 & 4.2 & 4.1 & 3.5 & 4.7 & 4.0 & 5.1 & 3.2 & 4.3 \\
\hline Desv std & 0.2 & 0.1 & 0.2 & 0.2 & 0.1 & 0.2 & 0.1 & 0.2 & 0.1 & 0.2 \\
\hline \multicolumn{11}{|l|}{ Proteína, \% } \\
\hline Max & 3.3 & 3.3 & 2.9 & 3.0 & 3.1 & 3.0 & 3.0 & 3.1 & 2.9 & 3.0 \\
\hline Min & 3.1 & 3.0 & 2.7 & 2.9 & 3.0 & 2.9 & 2.8 & 2.9 & 2.8 & 2.6 \\
\hline$\% \mathrm{CV}$ & 1.7 & 3.6 & 3.2 & 1.1 & 2.2 & 1.5 & 2.1 & 1.8 & 2.2 & 5.7 \\
\hline Desv std & 0.1 & 0.1 & 0.1 & 0.0 & 0.1 & 0.0 & 0.1 & 0.1 & 0.1 & 0.2 \\
\hline
\end{tabular}

y sus coeficientes de variación no fueron superiores al $7.9 \%$ (Tabla 3 ). Como se observa en la figura 3 las variaciones en producción de leche estuvieron asociadas en menor grado a las variaciones en la oferta $\left(R^{2}=0.45, p<0.01\right)$ y en mayor grado a las variaciones en el consumo de forraje $\left(R^{2}=0.54 p>0.01\right)$ como lo refleja la figura 4. De la misma manera pero con un grado de asociación mucho menor, las variaciones en la concentración de proteína fueron explicadas por las variaciones en la oferta $\left(R^{2}=0.17, p<0.01\right)$ y en el consumo de forraje $\left(R^{2}=0.34\right.$, $\left.p<0.01\right)$. Las variaciones en el tenor de grasa de la leche no estuvieron asociadas con las variaciones en la oferta o en el consumo.

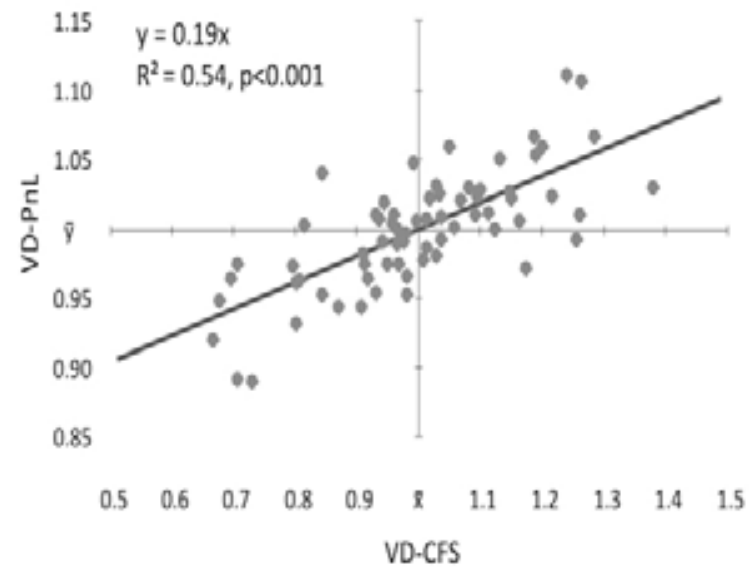

Figura 4. Efecto de la variación diaria del consumo de forraje sobre el volumen de leche. 


\section{DISCUSIÓN}

El objetivo principal de este trabajo fue determinar la magnitud de la variación de la oferta forrajera a través de los días en sistemas comerciales de producción de leche y determinar el efecto que estas tenían sobre el consumo de MS y el volumen y calidad de la leche.

Claramente se percibieron diferencias entre las variaciones de acuerdo al hato y periodo observado (Tabla 3 ) las cuales se pueden atribuir al manejo diferenciado que se practica de acuerdo al entorno expuesto.

\section{Capacidad de ajuste empírica y} variaciones de la oferta forrajera. La capacidad para entregar una oferta constante al animal (kgMS/vaca/d) obedece a distintos factores que van desde la facultad de producir una adecuada cantidad de biomasa, hasta lograr estimar la cantidad de área requerida para suministrar a los animales el alimento necesario para alcanzar su potencial productivo.

En la gran mayoría de las operaciones lecheras comerciales, es común encontrar que la oferta diaria de forraje es estimada de forma empírica, sin el apoyo de herramienta alguna que permita una medición objetiva de sus valores. En los hatos estudiados, las áreas asignadas a los animales con la cuerda eléctrica mostraron asociación débil con la disponibilidad de forraje por unidad de área $\left(R^{2}=0.27\right)$. La asociación entre estas variables fue inversa, lo que implica que a mayor disponibilidad de forraje el área asignada fue menor, sugiriendo que los ajustes se hicieron para mantener una oferta por animal más o menos constante. La precisión con que estos ajustes fueron realizados a nivel de campo fue baja como, lo indica una alta dispersión de los datos alrededor de la curva de tendencia. La mayor dispersión entre los valores observados (Figura 1b) se presentaron cuando la disponibilidad de forraje era menor, sugiriendo que en estas condiciones se presenta una mayor dificultad para estimar la disponibilidad de forraje o estimar las áreas a asignar.
La imprecisión en el ajuste de la cuerda entre un día y otro ocasionó variaciones en las ofertas de forraje (\%CV=8.3-37.5) dentro de los hatos en los periodos analizados. Los valores máximos y mínimos encontrados mostraron que en los hatos se presentaron días con valores sensiblemente menores o mayores al promedio.

Las variaciones en la oferta de forraje encontradas en los hatos sugieren que si el propósito de la asignación de la cuerda es mantener una oferta constante en periodos cortos de tiempo esto no se logra, dando lugar a situaciones de subestimación y/o sobreestimación de las áreas requeridas de oferta.

Variación en las ofertas y uso de la pradera. A pesar de las variaciones en las ofertas de forraje entre días y hatos, el tipo, estructura y manejo de las pasturas, e incluso entre características del manejo alimenticio, se encontró una expresión general de tipo curvilíneo (Figura 2), en la que el consumo (kgMS/d) y la utilización (\%) del forraje son determinados por la cantidad de oferta. Este tipo de relación curvilínea ha sido descrita por otros autores $(7,12-14)$ y sugeriría que la variación diaria en la oferta no modifica sustancialmente el principio general encontrado en las pasturas donde a mayor oferta hay un mayor consumo de forraje y un menor uso de la pastura. Por lo tanto, las variaciones diarias en oferta implicarían usos desiguales de las franjas de una pastura entre un día y otro. En los sistemas de manejo donde solo usan cuerda adelante del corte estas diferencias entre un día y otro no serían muy importantes pues en casos de mayores ofertas y por lo tanto mayor forraje residual, este quedaría para uso de las vacas en el siguiente día. Sin embargo, en aquellos sistemas donde usan doble cuerda (adelante y atrás) implicaría usos desiguales de la pradera y consecuentemente rebrotes también desiguales, dificultando el manejo de la cuerda en pasturas no uniformes.

Impacto de las variaciones en la oferta de forraje sobre el consumo de forraje, el volumen y la calidad de la dieta. El impacto que las variaciones diarias en la 
oferta tuvieron sobre el consumo de forraje y a su vez de este sobre la producción de leche corrobora lo encontrado por otros autores en condiciones de ofertas controladas. Por ejemplo, Bargo et al (4) encontraron que el consumo de MS de vacas lecheras sin suplementar aumentó de $17.7 \mathrm{~kg} / \mathrm{d}(2.9 \% \mathrm{PV})$ a $20.5 \mathrm{~kg} / \mathrm{d}(3.4 \% \mathrm{PV})$ cuando la oferta de forraje aumentó de 25 a $40 \mathrm{kgMS} / \mathrm{vaca} / \mathrm{d}$. Localmente, Mojíca et al (15) encontraron un incremento considerable en el consumo de pasto kikuyo en la Sabana de Bogotá cuando la oferta de forraje estuvo entre 2.5 y $4.0 \%$ PV.

En el presente estudio, la asociación entre consumo y oferta sugiere que por cada $\pm 10 \%$ de cambio diario en la oferta forrajera, el consumo de forraje variará en $\pm 7 \%$. Lo anterior sugiere que bajo los rangos de oferta encontrados en este estudio, los animales poseen una baja capacidad para adaptarse a los cambios en la oferta y por lo tanto su consumo está estrechamente relacionado con los cambios en la misma.

La literatura reporta diferentes valores de oferta por debajo de la cual el consumo se ve restringido, es decir, valores en los cuales la oferta determina el consumo de los animales. En su revisión de trabajos basados en oferta forrajera, Bargo et al (7) sugieren que para alcanzar el máximo consumo en vacas en pastoreo sin suplementación se debe dar una oferta entre tres y cinco veces el consumo esperado y para vacas lactantes suplementadas recomiendan una oferta de dos veces el consumo esperado o alrededor de $25 \mathrm{kgMS} / \mathrm{vaca} / \mathrm{d}$. En el presente estudio, la mayoría de los promedios de oferta en los periodos muestreados estuvieron por debajo de $25 \mathrm{~kg} / \mathrm{vaca} / \mathrm{d}$, solo en algunos casos se presentaron ofertas superiores donde aparentemente el máximo de consumo se alcanzó con valores un poco mayores a lo sugerido por Bargo et al (4). Esto indica que en la mayoría de los casos el consumo de forraje estuvo definido por la oferta y que en condiciones comerciales las variaciones en la oferta tienen una influencia directa sobre el consumo de forraje y por tanto la ingesta de nutrientes.
La importancia de las variaciones en el consumo de forraje debido a las variaciones en la oferta en las explotaciones comerciales explican a su vez la estrecha relación de estas con la producción de leche $(r=0.54$, $p<0.01$ ), y a pesar que el coeficiente de variación en la producción de leche fue mucho menor que los presentados por la oferta o el consumo. En este estudio, la producción se incrementa en un promedio de $0.9 \mathrm{~kg}$ por cada $\mathrm{kg}$ MS ingerido, consistente con lo hallado por Peyraud et al (16) y Ribeiro Filho et al (17) quienes encontraron en ensayos controlados un incremento aproximado de $1 \mathrm{~kg}$ de leche por cada $\mathrm{kgMS}$ ingerido. Otros autores también han descrito una clara asociación entre la oferta forrajera, el consumo de forraje y la producción de leche $(1-6,12-$ $15,18)$ o entre el consumo de energía y la producción de leche (19). Es interesante observar que esta dependencia entre consumo y producción de leche estuvo presente aún en periodos muy cortos de tiempo (un día a otro) y que por lo tanto la producción de leche a nivel comercial resultó ser especialmente sensible al manejo diario de la cuerda eléctrica y/o al manejo de la oferta forrajera. Por lo tanto, la medición de la biomasa disponible y de las áreas a asignar debería ser una tarea de especial cuidado en estos sistemas de producción.

El impacto de las variaciones diarias en la oferta de forraje y o consumo de forraje sobre la concentración de proteína en la leche fueron menores. Sin embargo, se observó que al aumentar la oferta de forraje en $1 \mathrm{~kg}$ de MS, la proteína se incrementaba en $0.2 \mathrm{~g} / \mathrm{kg}$ de leche producido $\left(R^{2}=0.21\right.$, $\mathrm{p}>0.01$ ), y que por cada kgMS de forraje consumido, la deposición de proteína aumentará cerca de $0.6 \mathrm{~g} / \mathrm{kg}$ de leche.

Aunque varios autores señalan que mayores ofertas de forraje o consumos de energía las concentraciones de proteína mejoran $(1,15,18,20)$ la menor asociación entre las variaciones el consumo y las variaciones en proteína $\left(R^{2}=0.34, p<0.01\right)$ sugeriría que este factor es más resistente a las variaciones en el consumo de nutrientes y por lo tanto explicaría los menores 

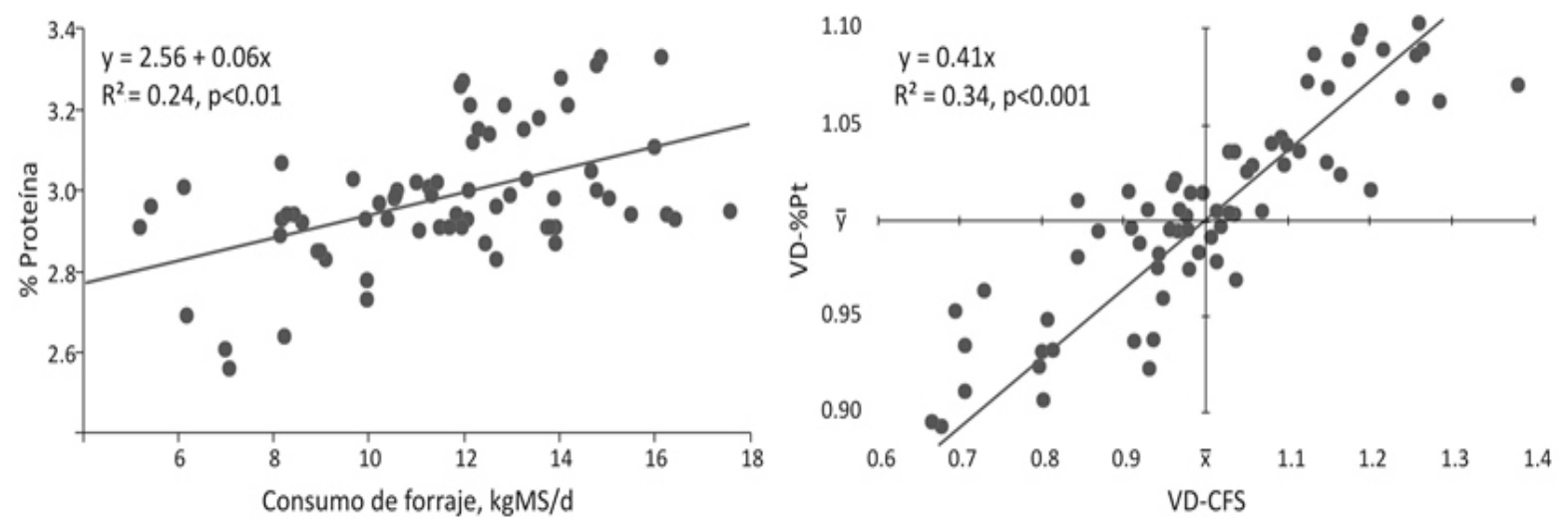

Figura 5. Efecto de la variación diaria del consumo de forraje sobre la proteína.

coeficientes de variación entre un día y otro. Sin embargo, el manejo de la cuerda tendría también un impacto aunque menor sobre las concentraciones de proteína en la leche y las variaciones que esta tiene entre un día y otro.

La concentración de grasa no presentó asociación alguna frente a las variaciones de la oferta de forraje o el consumo de forraje. La ausencia de respuesta en el contenido de grasa de la leche en función a la cantidad de oferta ya ha sido reportada por diversos autores $(15,18)$. No obstante, la proporción de forraje consumido en las dietas en relación con el concentrado suministrado mostró un efecto directo sobre el contenido de grasa en la leche $\left(R^{2}=0.52, p<0.001\right)$ (Figura 5) Whitlock et al, (21) reportaron tendencias similares, como al aumentar la proporción de concentrado en la ración disminuyen las concentraciones de grasa en leche y se ha reportado que en la medida que la suplementación de granos ricos en almidones aumenta la concentración de grasa disminuye $(4,22)$. Por lo tanto, los cambios en las relaciones entre forraje y concentrado debido a los cambios diarios en la oferta podrían relacionarse a las menores variaciones que se presentaron en las concentraciones de grasa en este estudio.

En conclusión, los datos encontrados en cinco hatos comerciales de la Sabana de Bogotá demostraron que los actuales criterios de estimación y suministro del forraje disponible en explotaciones locales de lechería especializada conllevan a un comportamiento variable en la oferta forrajera a través de los días. Esta variación explicó en gran medida la variación diaria del consumo de forraje y a su vez las variaciones en el consumo explicaron parcialmente las variaciones en la producción de leche. A pesar de lo anterior, los valores de producción y calidad de la leche presentaron fluctuaciones diarias menores que las de la oferta o el consumo. La grasa y la proteína presentaron una baja relación con las variaciones en la oferta o el consumo de forraje.

De acuerdo a lo expuesto, las oscilaciones en la cantidad de oferta forrajera no son suficientes para explicar las variaciones diarias en la composición de la leche. Sin embargo, el contenido de proteína mostró mayor grado de asociación con el consumo de forraje, mientras el tenor graso tuvo un alto grado de asociación con la proporción de forraje dentro de la ración.

\section{Agradecimientos}

A los propietarios de los hatos involucrados, y a la Asociación de Ganaderos de Facatativá, Alex Gutiérrez por su colaboración en la logística y enlace con el Laboratorio de Calidad de Colanta (Funza). A Constanza Riveros por su apoyo en el Laboratorio de Nutrición Animal de la Facultad de Medicina Veterinaria y de Zootecnia de la Universidad Nacional de Colombia - Sede Bogotá. 


\section{REFERENCIAS}

1. O'Brien B, Murphy JJ, Connolly JF, Mehra RAJ, Guinee TP, Stakelum G. Effect of altering the daily herbage allowance in mid lactation on the composition and processing characteristics of bovine milk. J Dairy Res 1997; 64:621-666.

2. O'Brien B, Dillon P, Murphy JJ, Mehra RK, Guinee TP, Connolly JF et al. Effects of stocking density and concentrate supplementation of grazing dairy cows on milk production composition and processing characteristics. J Dairy Res 1999; 66:165-176.

3. Auldist MJ, Thomson NA, Mackle TR, Hill JP, Prosser CG. Effects of pasture allowance on the yield and composition of milk from cows of different $\beta$ lactoglobulin phenotypes. J Dairy Sci 2000; 83:2069-2074.

4. Bargo F, Muller LD, Delahoy JE, Cassidy TW. Milk response to concentrate supplementation of high producing dairy cows grazing at two pasture allowances. J Dairy Sci 2002; 85:1777-1792.

5. Escobar A, Carulla JE. Efecto de la oferta forrajera sobre los parámetros productivos y composicionales de la leche en la Sabana de Bogotá. Rev Col Cienc Pec 2003; 16(Supl):67.

6. Blaser RE, Hammes RC, Fontenot JP, Bryant HT, Polan CE, Wolf DD et al. Principles in planning forage-animal systems. In: Blaser RE y Holliman MC, editors. Forage-Animal management systems. Virginia Agricultural Experiment Station Bulletin 86-7. Blacksburg (VA): Virginia Polytechnic Institute; 1986.

7. Bargo F, Muller LD, Kolver ES, Delahoy JE. Invited review: Production and digestion of supplemented dairy cows on pasture. J Dairy Sci 2003; 86:1-42.
8. Haydock KP, Shaw NH. The comparative yield method for estimating dry matter yield of pasture. Aust J Exp Agric 1975; 15:663-670.

9. Association of Official Analytical Chemists. Official methods of analysis of the association of official analytical chemists. 18th ed. Arlington, (VA): AOAC; 2005.

10. National Research Council. Nutrient requirements of dairy cattle. 7th ed. Washington DC (VA): National Academy Press; 2001.

11. R Development Core Team. R: A language and environment for statistical computing reference index version 2.10.0. $\mathrm{R}$ Foundation for Statistical Computing Vienna Austria. 2009. [Octubre 2009] URL: http:// www.R-project.org

12. Maher J, Stakelum G. and Rath M. Effect of daily herbage allowance on the performance of spring-calving dairy cows. Irish Journal of Agricultural Research 2003; 42:229-241.

13. Peyraud JL, Delaby L. Combiner la gestion optimale du pâturage et les performances des vaches laitières: enjeux et outils. INRA Prod Anim $2005 ; 18(4): 231-240$.

14. Taweel HZ. Improving dry-matter intake of perennial-ryegrass pasture by dairy cows. In: Elgersma A, Dijkstra $\mathrm{J}$ and Tamminga $\mathrm{S}$, editors. Fresh herbage for dairy cattle. Wageningen: Wageningen Press; 2006.

15. Mojíca JE, Castro E, León J, Cárdenas EA, Pabón ML y Carulla JE. Efecto de la oferta de pasto kikuyo (Pennisetum clandestinum) sobre la producción y calidad composicional de la leche bovina. Livestock Research for Rural Development 2009; 21(1):1-12. 
16. Peyraud JL, Delagarde R, Delaby L. Relationships between milk production grass dry matter intake and grass digestion. In: Proceedings of the 11th and 12th Irish Grassland Association Dairy Conference. Cork: Irish Grassland Association; 2001.

17. Ribeiro Filho HMN, Delagarde $\mathrm{R}$, Peyraud JL. Herbage intake and milk yield of dairy cows grazing perennial ryegrass swards or white clover/ perennial ryegrass swards at low- and medium-herbage allowances. Anim Feed Sci Technol 2005; 119:13-27.

18. Wales WJ, Doyle PT, Stockdale CR, and Dellow DW. Effects of variations in herbage mass, allowance, and level of supplement on nutrient intake and milk production of dairy cows in spring and summer. Aust J Exp Agr 1999; 39:119-130.
19. Coulon JB, Rémond $B$. Variations in milk output and milk protein content in response to the level of energy supply in the dairy cow: A review. Liv Prod Sci $1991 ; 29: 31-47$.

20. Whitlock LA, Schingoethe DJ, Hippen AR, Kalscheur KF and Abu Ghazaleh AA. Milk production and composition from cows fed high oil or conventional corn at two forage concentrations. J Dairy Sci 2003; 86:2428-2437.

21. Sanh MV, Wiktorsson H, Ly LV. Effects of natural grass forage to concentrate ratios and feeding principles on milk production and performance of crossbreed lactating cows. Asian Austral J Anim 2002; 15:650-657. 